\title{
Influence of the effective mass on the relative neutron density distribution inside a large sample in prompt-gamma neutron activation analysis
}

\author{
M. Blaauw,* I. H. Degenaar, J. J. M. de Goeij \\ Reactor Institute Delft, Faculty of Applied Sciences, University of Technology Delft, Mekelweg 15, 2629JB Delft, The Netherlands
}

(Received April 13, 2006)

\begin{abstract}
A Monte Carlo study was carried out to determine the influence of the effective scattering mass $\left(M_{e}\right)$ of the atoms on the neutron density profile inside and outside the sample illuminated by a thermal neutron beam as in large-sample prompt-gamma neutron activation analysis (LS-PGNAA). From theory it is known that the spatial neutron density distribution $(n(r))$ inside a large sample is not the same for atoms with the same macroscopic scattering and absorption cross-section $\left(\Sigma_{s}\right.$ and $\left.\Sigma_{a}\right)$ but different $M_{e}$, due to anisotropic scattering at low $M_{e}$. The probability of neutron absorption in the sample was found to be the same for materials with equal $\Sigma_{s}$ and $\Sigma_{a}$ but different $M_{e}$, even though the neutron density distribution in the sample was found to change slightly. In view of typical sample, collimator and detector dimensions, it is concluded that $M_{e}$ does not need to be taken into account in a correction method for neutron self-shielding in LS-PGNAA.
\end{abstract}

\section{Introduction}

In large-sample prompt-gamma neutron activation analysis (LS-PGNAA), the sample is larger than the neutron beam, say 1 liter and up, and extensive selfshielding effects occur. In a preceding paper, ${ }^{1}$ a method is presented to derive the macroscopic scattering and absorption cross sections, $\Sigma_{s}$ and $\Sigma_{a}$, by monitoring the neutron-density distribution $n(r)$ outside a large homogeneous sample material, in order to be able to perform quantitative LS-PGNAA, with an acuracy of better than $10 \%$. Another parameter that is likely to affect $n(r)$ in a large sample is the effective mass $\left(M_{e}\right)$ of the atoms in the sample material. In short, $M_{e}$ is a parameter to describe the atom mass $(M)$ and its associated chemical binding in one parameter so that the atoms can be treated as free gas atoms. When atoms can be treated as free gas atoms, i.e., neglecting the interaction between atoms in a molecule, the physics becomes easier and thus the calculation time in simulations becomes shorter.

In the study in the preceding paper, ${ }^{1}$ the atoms in the sample material were simulated as if they were rigidly bound. To check the validity of the approach, especially for light atoms inside a light molecule, a study was carried out on the influence of $M_{e}$ on $n(r)$ in two ways.

First, the direct influence of $M_{e}$ on $n(r)$ was investigated. Second, the influence was studied of $M_{e}$ on the flux monitors, used to subsequently determine the $\Sigma_{s}$ and $\Sigma_{a}$ of the sample material. Both the "true" and the "derived" $\Sigma_{s}$ and $\Sigma_{a}$ are used to calculate $n(r)$ and the $n(r)$ compared. This comparison was performed with respect to the total number of neutrons absorbed in the sample, the positions of the center of mass of the neutron density distributions in the sample, and the width of the neutron distributions.

\section{Theory}

The physical model used for the scattering process was the free-gas model, ${ }^{2}$ i.e., energy exchange between the neutrons and the scattering atoms was accounted for, as well as anisotropy of the scattering and thermal motion of the scattering atoms. The effective mass of the scattering atom, the temperature of the scatterer and the velocity distribution of the neutrons therefore play a part in this model.

The free-gas model is an imperfect model for neutron scattering by solids and liquids, commonly applied when the complete scattering kernel is unknown. However, it is appropriate for the current experiments, where neutrons colder than the sample as well as scattering by $\mathrm{H}_{2} \mathrm{O}$ play a part. The simpler alternative, based on the isotropic, no-energy-exchange model, can only be employed when the neutrons are not thermalized by the sample and the scattering can be considered to be isotropic in the laboratory system.

In our in-house software BUDA, ${ }^{3}$ the free-gas model is implemented, in part using the same Monte Carlo techniques as applied in MCNP. ${ }^{4}$ In this program, generalized neutron self-shielding factors $f$ are calculated defined by:

$$
f=\frac{R V}{\sum_{a, 0} N v_{0} n V}
$$

where $R$ is the capture rate density, $\mathrm{m}^{-3} \cdot \mathrm{s}^{-1} ; V$ is the sample volume, $\mathrm{m}^{3} ; \Sigma_{a, 0}$ is the macroscopic absorption cross section at $v_{0}, \mathrm{~m}^{2} ; v_{0}$ is the reference neutron velocity, $\mathrm{m} \cdot \mathrm{s}^{-1}$; conventionally taken as $2200 \mathrm{~m} \cdot \mathrm{s}^{-1} ; n$ is the neutron density, $\mathrm{m}^{-3}$, and $N$ the atom density, $\mathrm{m}^{-3}$. 


\section{Experimental}

\section{Influence of $M_{e}$ on $n(r)$ inside the sample material}

The simulations were carried out using the Monte Carlo code BUDA. ${ }^{3}$ Nine hypothetical sample materials with different $M_{e}$ were applied in the simulation. The macroscopic total cross section $\left(\Sigma_{t}\right)$ was varied from $0.028 \mathrm{~cm}^{-1}$ to $0.139 \mathrm{~cm}^{-1}$, i.e., the transmission $(T)$ of the $10-\mathrm{cm}$ thick sample [Eq. 2] varied from to 0.75 , to 0.25. In Eq. 2, $x$ is the thickness of the sample of $10 \mathrm{~cm}$ :

$$
T=e^{-\Sigma_{t} x}
$$

At each value of $\Sigma_{t}$, the macroscopic scattering cross section over macroscopic total cross section ratio $\left(\Sigma_{s} / \Sigma_{t}\right)$ was set to $0.25,0.50$ and 0.75 . The effective mass $\left(M_{e}\right)$ of the atoms in the sample material was set to $1,2,3,5$, $10,20,100$ and $1000 \mathrm{amu}$. The values of $\Sigma_{t}$ and $\Sigma_{s}$ are chosen between the values of air and sand and in the area where the approach described in the preceding paper ${ }^{1}$ was found to be applicable. Otherwise, the same experimental arrangement was modeled as in the preceding paper, that is, a 1-liter Teflon ${ }^{\mathrm{TM}}$ bottle illuminated by a thermal, $2.54 \mathrm{~cm}$ diameter neutron beam.

\section{Influence of $M_{e}$ on the determination of $\Sigma_{s}$ and $\Sigma_{a}$ of the sample material}

The influence of $M_{e}$ outside the sample material was determined by observing the probability $(P)$ of absorbing a neutron in four copper foils positioned around the sample. As described in the preceding paper, these $P$ 's are used in LS-PGNAA to derive the $\Sigma_{s}$ and $\Sigma_{a}$ of the sample material. If $M_{e}$ is influencing $P$ of one of the copper foils a change can be expected in the derived values of $\Sigma_{s}$ and $\Sigma_{a}$, leading to a change in the assumed $n(r)$ inside the sample material and consequently in the final element mass fractions.

In the preceding paper, $M_{e}$ of the sample material was set to $1000 \mathrm{amu}$ in all simulations. In this investigation it was tested if the method described there is also valid for sample materials with $M_{e}$ equal to 1,5 and 10. To that end, true values for $\Sigma_{s}$ and $\Sigma_{a}$ were selected, the simulation performed, derived values for $\Sigma_{s}$ and $\Sigma_{a}$ obtained from the copper $P$ 's as if $M_{e}$ was $1000 \mathrm{amu}$, and then used in a new simulation where $M_{e}$ was set to $1000 \mathrm{amu}$.

Three parameters were determined to test the effect of the error due to $M_{\text {eff }}$ in the determination of $\Sigma_{s}$ and $\Sigma_{a}$ on $n(r)$ inside the sample material. These were in order of importance: the average neutron density in the sample material (represented by the generalized self-shielding factor $f$ ), the $d$ coordinate of the centre of mass $\left(d_{C M}\right)$ of $n(r)$ and the average neutron distance from that centre of mass $(\Delta r$ and $\Delta d)$.
The $d_{C M}$ parameter was calculated with Eq. 2 . The average neutron distance from the center of mass in the $d$ direction, $\Delta d$, was calculated with Eq. 1 . The value for $\Delta r$ can be calculated with the same equation in which $d$ is replaced by $r$ :

$$
\begin{gathered}
d_{C M}=\frac{\sum_{v} n_{a} d}{\sum_{v} n_{a}} \\
\Delta d=\frac{\sum_{v} n_{a}\left|d-d_{C M}\right|}{\sum_{v} n_{a}}
\end{gathered}
$$

where $n_{a}$ is the total number of neutrons absorbed; $d$ is the depth of the position where the neutron absorption occurs from the point where the beam enters the sample material, $m ; \Delta d$ is the average neutron distance to $\mathrm{CM}$ in the d-direction, $m$, and $v$ is the voxel index number.

With parameters $d_{C M}, \Delta d$ and $\Delta r$ it can be shown whether the shape and position of of $n(r)$ are properly reproduced, the relevant aspect being the part of $n(r)$ seen by the detector, and the detector and collimator being placed at the positive $X$-axis.

\section{Results and discussion}

\section{Influence of $M_{e}$ on $n(r)$ inside the sample material}

Self-shielding factors $f$ of all simulated sample materials, as computed by BUDA, are given in Table 1 . As can be seen, the self-shielding factor $f$ for sample material with the same $\Sigma_{s} / \Sigma_{t}$ ratio and the transmission factor $T$ changed as $M_{e}$ changed, but by less than $3 \%$. This might seem to imply that the final mass fractions obtained with LS-PGNAA would also change less than $3 \%$ in first-order approximation, but this would only be true if the neutron densities observed by the flux monitors would remain unaffected.

\section{Influence of $M_{e}$ on the determination of $\Sigma_{s}$ and $\Sigma_{a}$ of the sample material}

The $\Sigma_{s}$ and $\Sigma_{a}$ derived from the copper $P$ 's with the method of the preceding paper can be seen in Table 2 for different sample materials as a function of $M_{e}$. In some sample materials the difference between the derived and the true input values for $\Sigma_{s}$ and $\Sigma_{a}$ is larger than $10 \%$. The largest deviations are found, as expected, when $\Sigma_{s}$ and $\Sigma_{a}$ of sample material with $M_{e}=1$ are determined.

With the true as well as with the derived $\Sigma_{s}$ and $\Sigma_{a}$ shown in Table 2, neutron density distributions $n(r)$ inside the sample were simulated and the differences between the "true" and "derived" $d_{C M}, \Delta d, \Delta r$ and $f$ determined $\left(d_{C M}, \Delta d\right.$ and $\Delta r$ are defined in the preceding paper). The results are shown in Tables 3, 4, 5 and 6 . 
It can be seen that the differences in $f$ are smaller than $4.7 \%$, on the average $1.5 \%$. This means that the final mass fractions obtained with LS-PGNAA will also be off by less than $4.7 \%$ in first-order approximation. When $M_{e}$ is 5 or larger the difference in $f$ is smaller than $1.8 \%$, on the average $1.0 \%$.

Table 1. Self-shielding factors for all simulated samples for different $M_{e}$

\begin{tabular}{|c|c|c|c|c|c|c|c|c|c|c|}
\hline \multicolumn{2}{|c|}{$\Sigma_{s} / \Sigma_{t}$} & 0.75 & 0.75 & 0.75 & 0.50 & 0.50 & 0.50 & 0.25 & 0.25 & 0.25 \\
\hline$M_{e}$ & $T$ & 0.75 & 0.50 & 0.25 & 0.75 & 0.50 & 0.25 & 0.75 & 0.50 & 0.25 \\
\hline & 1 & 0.0316 & 0.0309 & 0.0291 & 0.0302 & 0.0279 & 0.0243 & 0.0289 & 0.0255 & 0.0208 \\
\hline & 2 & 0.0315 & 0.0307 & 0.0289 & 0.0302 & 0.0278 & 0.0241 & 0.0289 & 0.0254 & 0.0207 \\
\hline & 3 & 0.0315 & 0.0306 & 0.0287 & 0.0301 & 0.0278 & 0.0241 & 0.0289 & 0.0254 & 0.0207 \\
\hline & 5 & 0.0314 & 0.0306 & 0.0286 & 0.0301 & 0.0277 & 0.0240 & 0.0289 & 0.0253 & 0.0206 \\
\hline & 10 & 0.0314 & 0.0305 & 0.0285 & 0.0301 & 0.0276 & 0.0239 & 0.0289 & 0.0253 & 0.0206 \\
\hline & 20 & 0.0313 & 0.0304 & 0.0284 & 0.0300 & 0.0276 & 0.0238 & 0.0288 & 0.0253 & 0.0206 \\
\hline & 00 & 0.0313 & 0.0303 & 0.0283 & 0.0300 & 0.0276 & 0.0238 & 0.0288 & 0.0253 & 0.0205 \\
\hline 10 & & 0.0313 & 0.0303 & 0.0283 & 0.0300 & 0.0276 & 0.0238 & 0.0288 & 0.0253 & 0.0205 \\
\hline
\end{tabular}

The imprecision due to counting statistics in the number of absorbed neutrons and thus in $f$ was smaller than $0.01 \%$.

Table 2. True and derived $\Sigma_{s}$ and $\Sigma_{a}$ for different sample materials as a function of $M_{e}$

\begin{tabular}{ccccccc}
\hline$\Sigma_{s} / \Sigma_{t}$ & $T$ & & True & $M_{e}=1$ & $M_{e}=5$ & $M_{e}=10$ \\
\hline 0.75 & 0.75 & $\Sigma_{a}$ & $7.19 \cdot 10^{-3}$ & $8.06 \cdot 10^{-3}$ & $7.21 \cdot 10^{-3}$ & $7.68 \cdot 10^{-3}$ \\
& & $\Sigma_{s}$ & $2.16 \cdot 10^{-2}$ & $3.08 \cdot 10^{-2}$ & $2.43 \cdot 10^{-2}$ & $2.27 \cdot 10^{-2}$ \\
0.75 & 0.50 & $\Sigma_{a}$ & $1.73 \cdot 10^{-2}$ & $2.05 \cdot 10^{-2}$ & $1.86 \cdot 10^{-2}$ & $1.87 \cdot 10^{-2}$ \\
& & $\Sigma_{s}$ & $5.20 \cdot 10^{-2}$ & $7.03 \cdot 10^{-2}$ & $5.75 \cdot 10^{-2}$ & $5.43 \cdot 10^{-2}$ \\
0.75 & 0.25 & $\Sigma_{a}$ & $3.47 \cdot 10^{-2}$ & $3.97 \cdot 10^{-2}$ & $3.49 \cdot 10^{-2}$ & $3.58 \cdot 10^{-2}$ \\
& & $\Sigma_{s}$ & $1.04 \cdot 10^{-1}$ & $2.34 \cdot 10^{-1}$ & $1.15 \cdot 10^{-2}$ & $1.08 \cdot 10^{-2}$ \\
0.50 & 0.75 & $\Sigma_{a}$ & $1.44 \cdot 10^{-2}$ & $1.30 \cdot 10^{-2}$ & $1.42 \cdot 10^{-2}$ & $1.45 \cdot 10^{-2}$ \\
& & $\Sigma_{s}$ & $1.44 \cdot 10^{-2}$ & $2.27 \cdot 10^{-2}$ & $1.73 \cdot 10^{-2}$ & $1.59 \cdot 10^{-2}$ \\
0.50 & 0.50 & $\Sigma_{a}$ & $3.47 \cdot 10^{-2}$ & $3.17 \cdot 10^{-2}$ & $3.25 \cdot 10^{-2}$ & $3.47 \cdot 10^{-2}$ \\
& & $\Sigma_{s}$ & $3.47 \cdot 10^{-2}$ & $5.19 \cdot 10^{-2}$ & $4.16 \cdot 10^{-2}$ & $3.79 \cdot 10^{-2}$ \\
0.50 & 0.25 & $\Sigma_{a}$ & $6.93 \cdot 10^{-2}$ & $6.59 \cdot 10^{-2}$ & $6.56 \cdot 10^{-2}$ & $6.50 \cdot 10^{-2}$ \\
& & $\Sigma_{s}$ & $6.93 \cdot 10^{-2}$ & $9.43 \cdot 10^{-2}$ & $7.90 \cdot 10^{-2}$ & $7.71 \cdot 10^{-2}$ \\
0.25 & 0.75 & $\Sigma_{a}$ & $2.16 \cdot 10^{-2}$ & $2.11 \cdot 10^{-2}$ & $2.15 \cdot 10^{-2}$ & $2.31 \cdot 10^{-2}$ \\
& & $\Sigma_{s}$ & $7.19 \cdot 10^{-3}$ & $1.26 \cdot 10^{-2}$ & $9.77 \cdot 10^{-3}$ & $8.20 \cdot 10^{-3}$ \\
0.25 & 0.50 & $\Sigma_{a}$ & $5.20 \cdot 10^{-2}$ & $5.19 \cdot 10^{-2}$ & $5.40 \cdot 10^{-2}$ & $5.51 \cdot 10^{-2}$ \\
& & $\Sigma_{s}$ & $1.73 \cdot 10^{-2}$ & $2.78 \cdot 10^{-2}$ & $2.14 \cdot 10^{-2}$ & $1.96 \cdot 10^{-2}$ \\
0.25 & 0.25 & $\Sigma_{a}$ & $1.04 \cdot 10^{-1}$ & $1.09 \cdot 10^{-1}$ & $1.03 \cdot 10^{-1}$ & $1.09 \cdot 10^{-1}$ \\
& & $\Sigma_{s}$ & $3.47 \cdot 10^{-2}$ & $4.41 \cdot 10^{-2}$ & $4.18 \cdot 10^{-2}$ & $3.68 \cdot 10^{-2}$ \\
\hline & & & & & & \\
& & & & &
\end{tabular}

Table 3. Difference (in \%) in self-shielding factor $f$ for sample materials simulated with the derived and the true $\Sigma_{s}$ and $\Sigma_{a}$ as function of $M_{e}$

\begin{tabular}{|c|c|c|c|c|c|c|c|c|c|}
\hline$\Sigma_{S} / \Sigma_{t}$ & 0.75 & 0.75 & 0.75 & 0.50 & 0.50 & 0.50 & 0.25 & 0.25 & 0.25 \\
\hline \begin{tabular}{l|l}
$M_{e}$ & $T$
\end{tabular} & 0.75 & 0.50 & 0.25 & 0.75 & 0.50 & 0.25 & 0.75 & 0.50 & 0.25 \\
\hline 1000 & -0.8 & -1.2 & -1.8 & -0.1 & 0.1 & -0.4 & -1.2 & -1.4 & -1.1 \\
\hline 10 & -0.4 & -1.1 & -1.3 & -0.1 & -0.2 & 1.5 & -0.7 & -1.4 & -2.1 \\
\hline 5 & -0.2 & -1.1 & -0.3 & 0.1 & 0.9 & 0.9 & 0.1 & -1.0 & -0.1 \\
\hline 1 & -0.7 & -2.4 & -4.7 & 0.6 & 0.9 & -0.3 & 0.3 & -0.2 & -3.0 \\
\hline
\end{tabular}

The inaccuracy due to counting statistics is smaller than $0.01 \%$.

Table 4. Change in $\mathrm{CM}-d$ coordinate (in $\mathrm{cm}$ ), as function of $M_{e}$ for all sample materials simulated with the determined and the original $\Sigma_{s}$ and $\Sigma_{a}$

\begin{tabular}{|c|c|c|c|c|c|c|c|c|c|}
\hline$\Sigma_{s} / \Sigma_{t}$ & 0.75 & 0.75 & 0.75 & 0.50 & 0.50 & 0.50 & 0.25 & 0.25 & 0.25 \\
\hline$M_{e} \quad T$ & 0.75 & 0.50 & 0.25 & 0.75 & 0.50 & 0.25 & 0.75 & 0.50 & 0.25 \\
\hline 1000 & 0.00 & -0.01 & 0.00 & 0.00 & -0.01 & -0.01 & -0.01 & -0.03 & 0.00 \\
\hline 10 & -0.01 & -0.02 & -0.02 & -0.01 & -0.02 & -0.01 & -0.02 & -0.03 & 0.00 \\
\hline 5 & -0.01 & -0.03 & -0.04 & -0.01 & -0.02 & -0.02 & -0.02 & -0.04 & -0.03 \\
\hline 1 & -0.05 & -0.10 & -0.14 & -0.03 & -0.06 & -0.09 & -0.03 & -0.06 & 0.00 \\
\hline
\end{tabular}


Table 5. Change in the width of the neutron distribution in the $r$-direction (in $\mathrm{cm}$ ), as a function of $M_{e}$ for all sample materials simulated with the determined and the original $\Sigma_{s}$ and $\Sigma_{a}$

\begin{tabular}{|c|c|c|c|c|c|c|c|c|c|}
\hline$\Sigma_{s} / \Sigma_{t}$ & 0.75 & 0.75 & 0.75 & 0.50 & 0.50 & 0.50 & 0.25 & 0.25 & 0.25 \\
\hline \begin{tabular}{l|l}
$M_{e}$ & $T$ \\
\end{tabular} & 0.75 & 0.50 & 0.25 & 0.75 & 0.50 & 0.25 & 0.75 & 0.50 & 0.25 \\
\hline 1000 & -0.01 & -0.0 & -0.07 & -0.01 & -0.04 & -0.03 & 0.00 & -0. & -0.01 \\
\hline 10 & 10 & -0.0 & -0.0 & -0.01 & -0. & -0.03 & -0.01 & -0 . & -0.01 \\
\hline 5 & Q & & $-0,09$ & -0.0 & -0 . & -0 . & -0. & -0 . & -0.02 \\
\hline 1 & -0.01 & -0.06 & -0.10 & -0.01 & -0.04 & -0.05 & -0.01 & -0.02 & -0.01 \\
\hline
\end{tabular}

The inaccuracy due to counting statistics in the number of absorbed neutrons is smaller than $0.01 \%$.

Table 6. Change in the width of the neutron distribution in the $d$-direction (in $\mathrm{cm}$ ), as a function of $M_{e}$ for all sample materials simulated with the determined and the original $\Sigma_{s}$ and $\Sigma_{a}$

\begin{tabular}{|c|c|c|c|c|c|c|c|c|c|}
\hline$\Sigma_{s} / \Sigma_{t}$ & 0.75 & 0.75 & 0.75 & 0.50 & 0.50 & 0.50 & 0.25 & 0.25 & 0.25 \\
\hline \begin{tabular}{l|l}
$M_{e}$ & $T$
\end{tabular} & 0.75 & 0.50 & 0.25 & 0.75 & 0.50 & 0.25 & 0.75 & 0.50 & 0.25 \\
\hline 1000 & -0.01 & -0.01 & -0.02 & 0.00 & 0.01 & 0.00 & 0.00 & 0.01 & 0.00 \\
\hline 10 & 0.00 & -0.01 & -0.02 & 0.00 & 0.00 & 0.02 & 0.00 & 0.00 & 0.00 \\
\hline 5 & 0.00 & -0.01 & -0.01 & 0.01 & 0.02 & 0.02 & 0.01 & 0.01 & 0.01 \\
\hline 1 & 0.00 & -0.01 & -0.05 & 0.02 & 0.02 & 0.00 & 0.02 & 0.02 & -0.03 \\
\hline
\end{tabular}

The influence on the position of the center of mass of $n(\mathrm{r})$ of $0.14 \mathrm{~cm}$ at most is quite small as compared to reasonable collimator opening sizes and should be too small to be noticeable in the final element mass fractions. The maximum influence on $\Delta r(0.10 \mathrm{~cm})$ and $\Delta d(0.05 \mathrm{~cm})$ is also found to be quite small.

The largest differences are found with small $M_{e}$. Elements with atoms with $M_{e}$ smaller than 10 are $\mathrm{H}, \mathrm{He}$, $\mathrm{Li}$ and $\mathrm{Be}$. Future sample materials will not consist of $\mathrm{He}$ and $\mathrm{H}_{2}$, because these are gasses. In liquids and solids, the effective masses of these light atoms will be much higher than their atomic masses. The effective mass of hydrogen for thermal neutrons in water, for example, is about $2 \mathrm{amu}$, in polyethylene about $8 \mathrm{amu}$. In such materials, inaccuracies of perhaps $2.5 \%$ due to the effect studied in this paper could be expected. This is well within the design criteria of our LS-PGNAA system-to-be.

\section{Conclusions}

For all sample materials with the same $\Sigma_{s} / \Sigma_{t}$ ratio and $T$, but different $M_{e}$, the total number of neutrons absorbed in the sample material and the position of the CM of $n(r)$ are virtually equal.

When the $n(r)$ inside the sample material is simulated with the $\Sigma_{s}$ and $\Sigma_{a}$ derived with the method described in the preceding paper, ${ }^{1}$ the errors made in the average neutron density, the assumed position of the center of mass of the neutron distribution, and the widths of the neutron distribution $\Delta r$ and $\Delta d$ all depend on $M_{e}$. The average neutron density deviated on the average $1.5 \%$ at $M_{e}=1$; at $M_{e}=5$ and higher the deviations are smaller, on the average $0.9 \%$. The change in position of the center of mass $d_{C M}$ was $0.14 \mathrm{~cm}$ at most, the change in $\Delta r$ was $0.10 \mathrm{~cm}$ at most, and that of $\Delta d$ was $0.05 \mathrm{~cm}$ at most.

The contribution to the final error in the element mass fraction will be small. The error in $f$ propagates linearly to the mass fractions. The errors in parameters $d_{C M}, \Delta d$ and $\Delta r$ only have an effect through the gammaattenuation correction, and because the distance between the detector and the centre of mass of the sample will be $10 \mathrm{~cm}$ or more, and the collimator opening size is likely to be in the order of $\mathrm{cm}$, this contribution is expected to be negligible.

Summarizing, in practice no correction has to be made for $M_{e}$ of the atoms in the sample material in LSPGNAA. The free gas model can be applied in the methodology using a $M_{e}$ of $1000 \mathrm{amu}$ for all sample materials simulated, or equivalently, isotropic scattering in the laboratory system can be assumed.

\section{References}

1. M. Blaauw, I. H. Degenaar, J. J. M. De GoeiJ, J. Radioanal. Nucl. Chem., 271 (2007) 765.

2. H. A. BETHE, Elementary Nuclear Theory: A Short Course on Selected Topics, Wiley, New York, 1952.

3. M. BLAAUW, BUDA, A Program for Incoherent Neutron Scattering Monte Carlo Computations, Version 8, Reactor Institute Delft, The Netherlands, 2000.

4. J. BRIESMEISTER, MCNP: A General Monte Carlo N-particle Transport Code, Version 4B, Los Alamos National Laboratory, Los Alamos, 1997. 